\title{
Dynamical approach to Lévy processes
}

\author{
Paolo Allegrini, ${ }^{1}$ Paolo Grigolini, ${ }^{1,2,3}$ and Bruce J. West ${ }^{1}$ \\ ${ }^{1}$ Center for Nonlinear Science, University of North Texas, P.O. Box 5368, Denton, Texas 76203-5368 \\ ${ }^{2}$ Dipartimento di Fisica dell'Universitá di Pisa, Piazza Torricelli 2, 56100, Pisa, Italy \\ ${ }^{3}$ Istituto di Biofisica del Consiglio Nazionale delle Ricerche, Via S. Lorenzo 26, 56127 Pisa, Italy
}

(Received 3 June 1996)

\begin{abstract}
We derive the diffusion process generated by a correlated dichotomous fluctuating variable $y$ starting from a Liouville-like equation by means of a projection procedure. This approach makes it possible to derive all statistical properties of the diffusion process from the correlation function of the dichotomous fluctuating variable $\Phi_{y}(t)$. Of special interest is that the distribution of the times of sojourn in the two states of the fluctuating process is proportional to $d^{2} \Phi_{y}(t) / d t^{2}$. Furthermore, in the special case where $\Phi_{y}(t)$ has an inverse power law, with the index $\beta$ ranging from 0 to 1 , thus making it nonintegrable, we show analytically that the statistics of the diffusing variable approximate in the long-time limit the $\alpha$-stable Lévy distributions. The departure of the diffusion process of dynamical origin from the ideal condition of the Lévy statistics is established by means of a simple analytical expression. We note, first of all, that the characteristic function of a genuine Lévy process should be an exponential in time. We evaluate the correction to this exponential and show it to be expressed by a harmonic time oscillation modulated by the correlation function $\Phi_{y}(t)$. Since the characteristic function can be given a spectroscopic significance, we also discuss the relevance of our results within this context. [S1063-651X(96)08611-4]

PACS number(s): 02.50.Ey, 05.40.+j
\end{abstract}

\section{INTRODUCTION}

The basis of our understanding of statistical physics is the phenomenological theory of Brownian motion [1] and the chaotic behavior of nonintegrable Hamiltonian systems [2]. The statistics in Brownian motion arises from the coupling of the system of interest to the environment [3], resulting in erratic variations in the velocity of the diffusing particle. In the Hamiltonian case, however, the random fluctuations are an intrinsic property of the nonlinear system dynamics [4]. If a dynamical system such as a standard map is fully chaotic, meaning that all the Kolmogorov-Arnold-Moser tori have become globally unstable and disintegrated, producing a chaotic sea in phase space, then the average energy of the system increases linearly in time and the system is as diffusive as the Brownian particle. This relation between statistics and dynamics has been known for nearly two decades [5], but it is only recently that the connection has been systematically exploited to provide a dynamical basis for statistical physics. Bianucci et al. [6] have shown that statistical physics can be a consequence of the existence of chaotic solutions to lowdimensional nonlinear dynamical equations thereby blending the above two points of view.

Concurrently, explaining the phenomenon of anomalous diffusion in which the mean square amplitude of a process increases in time as $t^{2 H}$ with $H \neq 1 / 2$ has attracted the attention of a number of investigators. The description of such processes using nondiffusive Lévy statistics has been popular among a number of researchers [7-9]. This has led to studies to make the proper connection between Lévy stable processes and certain dynamical systems. One strategy is to use the fact that chaotic orbits stick to the cantori at the phase space boundary between stable islands and the chaotic sea in weakly chaotic systems [10]. Another strategy, adroitly implemented by Zumofen and Klafter [11], uses the frame- work of continuous-time random walks (CTRW's) to derive the Lévy stable process. This latter technique has also been directly applied to the case where the dynamical system is the standard map [12]. The final strategy that has been developed uses a master equation, independently of whether or not the system is Hamiltonian [13]. However, regardless of the strategy, no one has yet succeeded in deriving the $\alpha$-stable Lévy processes using a totally dynamical aprroach. As an example of totally dynamical approach to diffusion we have in mind the works of Refs. [14-16] and the more recent approach of Bianucci et al. [6]. These authors [6] derived a Fokker-Planck equation, namely, both the diffusion and the friction term from a microscopic Liouville equation with no statistical assumptions.

It is not only in the development of the foundations of statistical physics that one sees an interest in Lévy stable processes, but also in the foundations of relativistic quantum mechanics. Garbaczewski [17] maintains that determining the dynamical origins of Lévy processes may be of the same importance to relativistic quantum mechanics that the dynamical derivation of Brownian motion using path integrals had to nonrelativistic quantum mechanics. A relevant example of the latter case is given by Beck [18], who adopted the deterministic derivation of Brownian motion as a possible way of generalizing the nonrelativistic version of quantum mechanics.

It is not only transport that is affected by Lévy processes through anomalous diffusion, but relaxation processes as well. For example, the Maxwell-Zener standard constitutive equations relating stress to strain have been generalized to fractional order differential equations in time $[19,20]$. Glockle and Nonnenmacher [21] pointed out some relations of fractional differential equations to CTRW's of trapping type leading to the identification of the fractional order parameter with the index of the inverse power-law waiting- 
time distribution function and the Lévy index. The complex susceptibility for the relaxation process can be related to the Fourier transform of the characteristic function, which if the relaxation is exponential yields a relaxation time that is a power of the inverse wave number. The algebraic power of the wave number is just the Lévy index.

More generally the characteristic function is more closely tied to experimentally observable quantities than is the probability density. This brings us to our final example and the application developed in this paper, which is magnetic resonance in the presence of a long-range correlated process. We focus our attention on a dynamical process that in the longtime region becomes equivalent to an $\alpha$-stable Lévy process. The Hamiltonian of interest is

$$
\hat{H}=\omega_{0} \hat{\sigma}_{x}+g \hat{\sigma}_{z} \hat{y}+\hat{H}_{B},
$$

where $\hat{y}$ is a bath variable whose dynamics are generated by the Hamiltonian $\hat{H}_{B}$. This model is ubiquitous in physics and its applications range from magnetic resonance [22], exciton transport in solids [23] in the two-state version studied by Kenkre and co-workers [24] to macroscopic manifestations of quantum mechanics [25]. The usual assumption made in each of these applications is that the operator $\hat{y}$ is the sum of the displacements of the harmonic oscillators in the bath, so as to result, in the appropriate limit, in a classical stochastic variable having Gaussian statistics. Here we explore the consequences of modifying this approach in such a way that the statistics of the variable $y$ are non-Gaussian.

In the particular case when the natural frequency $\omega_{0}$ in (1) is set to zero the average time evolution of $\hat{\sigma}_{x}$ is given by

$$
\left\langle\hat{\sigma}_{x}(t)\right\rangle=\left\langle T \exp \left(\frac{2 i g}{\hbar} \int_{0}^{t} \hat{y}\left(t^{\prime}\right) d t^{\prime}\right)\right\rangle,
$$

where $T$ denotes the time ordering of the following exponential time integration and the displacement operator in the interaction representation is given by

$$
\hat{y}(t) \equiv e^{i \hat{H} t / \hbar} \hat{y} e^{-i \hat{H} t / \hbar} .
$$

If we now make the assumption that the displacement operator can be replaced by a corresponding classical variable, we can define the new variable

$$
x(t) \equiv \int_{0}^{t} y\left(t^{\prime}\right) d t^{\prime},
$$

so that the average (2) takes the form of a characteristic function

$$
\left\langle\hat{\sigma}_{x}(t)\right\rangle=\left\langle e^{-i k x(t)}\right\rangle,
$$

where the Fourier transform variable is given by

$$
k \equiv \frac{2 g}{\hbar} .
$$

Thus we see that the average time evolution of the $x$ component of the dipole operator corresponds to the characteristic function of the unperturbed process of diffusion of the dynamical variable $x(t)$. Equation (5) is the characteristic function because it is formally the Fourier transform of the probability density for the variable $x$

$$
\left\langle\hat{\sigma}_{x}(t)\right\rangle=\int e^{-i k x} P(x ; t) d x ;
$$

however, the probability density $P(x ; t)$, is, by assumption, not Gaussian. We are particularly interested in the cases where the statistics are Lévy stable. In Sec. II we develop the exact equation of evolution for a dichotomous process having correlated fluctuations. In Sec. III we study the case with no time-scale separation and we show that the resulting anomalous diffusion coincides, within a given approximation, with an $\alpha$-stable Lévy process. In Sec.IV the characteristic function is shown to separate into two pieces: one the standard exponential form in time for a Lévy process and the other a harmonically modulated inverse power law in time. Some details of the numerical method used are discussed in the Appendix.

\section{AN EXACT EQUATION FOR DIFFUSION RESULTING FROM A DICHOTOMOUS FLUCTUATING VARIABLE}

We have seen that the motion of the $x$ component of the dipole is equal to the characteristic function of the diffusion process $x$. Let us therefore focus our attention on the unperturbed diffusion process. This is described by the equation of motion

$$
\dot{x}(t)=y(t) .
$$

From a formal point of view it is convenient to adopt the Liouville-like picture

$$
\frac{\partial \rho(x, y, \mathbf{R} ; t)}{\partial t}=\left(-y \frac{\partial}{\partial x}+\hat{\Gamma}\right) \rho(x, y, \mathbf{R} ; t),
$$

where $\mathbf{R}$ denotes the set of additional variables necessary to define the dynamics of $y$. In the special case where we adopt a nonlinear map as a dynamical generator $[9,13]$, we can obtain to some extent an erratic behavior for $y$ without introducing additional variables. However, we want to keep our treatment at a level as general as possible. Thus we adopt the general notation of (9). We make only the simplifying assumption that $y$ is a dichotomous variable,

$$
y= \pm W .
$$

From a formal point of view we can imagine the classical variable $y$ as a sort of operator characterized by the two eigenstates $|+\rangle$ and $|-\rangle$,

$$
\hat{y}| \pm\rangle= \pm W| \pm\rangle .
$$

We do not set any bias, namely, we want to give the same statistical weight to the two states. Thus we assume the equilibrium state to be given by

$$
\left|p_{0}\right\rangle=\frac{1}{\sqrt{2}}(|+\rangle+|-\rangle)
$$

Formally this means that the application of $\hat{y}$ to the equilibrium state results in the "excited" state defined by 


$$
\left|p_{1}\right\rangle=\frac{\hat{y}\left|p_{0}\right\rangle}{W}
$$

In general, for a complete description of the dynamical process one has to consider more than two states. We write

$$
\langle y y(t)\rangle=\left\langle p_{0}\left|\hat{y} e^{\hat{\Gamma} t} \hat{y}\right| p_{0}\right\rangle=W^{2}\left\langle p_{1}\left|e^{\hat{\Gamma} t}\right| p_{1}\right\rangle .
$$

Due to the dichotomous nature of the variable $y$ we have

$$
\left\langle p_{1}\left|e^{\hat{\Gamma} t}\right| p_{1}\right\rangle=\sum_{\mu \neq 0}\left\langle p_{1} \mid \mu\right\rangle\left\langle\mu \mid p_{1}\right\rangle e^{-\Lambda_{\mu} t}
$$

where the $|\mu\rangle$ 's are eigenstates of the operator $\hat{\Gamma}$, with eingenvalue $\Lambda_{\mu}$, distinct from $\left|p_{0}\right\rangle$, which is the eigenstate with vanishing eigenvalue (the equilibrium state). Note that $|\mu=0\rangle=\left|p_{0}\right\rangle$. Within the framework of this quantumlike formalism, it is convenient to define

$$
\sigma_{\mu}(x, t)=\langle\mu \mid \rho(x, y, \mathbf{R} ; t)\rangle,
$$

with $\mu=0,1,2, \ldots$ We are interpreting the distribution $\rho$ of (16) as a sort of ket vector $|\rho\rangle$. By multiplying (9) on the left by the states $|\mu\rangle$ we get

$$
\begin{gathered}
\frac{\partial}{\partial t} \sigma_{0}(x ; t)=-W \sum_{\mu \neq 0}^{\infty} a_{\mu} \frac{\partial}{\partial x} \sigma_{\mu}(x ; t), \\
\frac{\partial}{\partial t} \sigma_{\mu}(x ; t)=-W a_{\mu}^{*} \frac{\partial}{\partial x} \sigma_{0}-\Lambda_{\mu} \sigma_{\mu}(x ; t),
\end{gathered}
$$

with $\mu=1,2, \ldots$, and $a_{\mu}=\left\langle\mu|\hat{y}| p_{0}\right\rangle$.

By solving the second equation and replacing the solution into the former equation, we get

$$
\frac{\partial}{\partial t} \sigma_{0}(x ; t)=W^{2} \sum_{\mu \neq 0}\left|a_{\mu}\right|^{2} \int_{0}^{t} d t^{\prime} e^{-\Lambda_{\mu}\left(t-t^{\prime}\right)} \frac{\partial^{2}}{\partial x^{2}} \sigma_{0}(x ; t) .
$$

From now on we shall focus on the reduced density matrix $\sigma_{0}(x ; t)$ and for the sake of simplicity we shall omit the subscript 0 . Using (14) and (15) we can rewrite (18) in the form

$$
\frac{\partial}{\partial t} \sigma(x ; t)=\int_{0}^{t} d t^{\prime}\left\langle y y\left(t-t^{\prime}\right)\right\rangle \frac{\partial^{2}}{\partial x^{2}} \sigma(x ; t) .
$$

In conclusion, in the dichotomous case we can express the time evolution of the probability distribution by means of an exact equation (19). This is a sort of generalization of the results obtained by Horstemke and Lefever [26] in the special case of an exponential correlation function.

Notice that if we integrate the equation of motion (8), under the assumption of stationarity, namely, that the correlation function $\left\langle y\left(t_{1}\right) y\left(t_{2}\right)\right\rangle$ depends only on $\left|t_{1}-t_{2}\right|$, we find

$$
\frac{d}{d t}\left\langle x^{2}(t)\right\rangle=2 \int_{0}^{t} d t^{\prime}\left\langle y y\left(t-t^{\prime}\right)\right\rangle+\left\langle x^{2}(0)\right\rangle,
$$

which coincides with the prescription that one one would obtain from (19). This is not surprising since both (19) and (20) are exact results.

\section{THE LÉVY PROCESS AS A TIME ASYMPTOTIC LIMIT}

Let us define now the normalized correlation function

$$
\Phi_{y}(t) \equiv \frac{\langle y y(t)\rangle}{\left\langle y^{2}\right\rangle}
$$

and assume that this function decays to zero without oscillations making them negative. In this condition, it makes sense to define the "microscopic" time scale

$$
\tau \equiv \int_{0}^{\infty} \Phi_{y}\left(t^{\prime}\right) d t^{\prime} .
$$

If this time scale exists and is a nonzero finite constant, it is straightforward to show, using the central limit theorem, that the diffusion process for $x$ taking place at times much larger than $\tau$ becomes indistinguishable from an ordinary Brownian diffusion.

The simplest possible way to extend the analysis beyond this ordinary condition, and so using the exact equation (19) to address nontrivial problems, is to assume

$$
\Phi_{y}(t)=\frac{A}{\left(A^{1 / \beta}+t\right)^{\beta}}, t \geqslant 0,
$$

with

$$
0<\beta<1 \text {. }
$$

Note that the form chosen for the correlation function makes it possible to fulfill the normalization condition

$$
\Phi_{y}(0)=1 .
$$

This condition (24) corresponds to a similar condition already examined in previous papers $[9,13]$. However, the property concerned the waiting-time distribution in one of the two states of the variable $y,|+\rangle$ and $|-\rangle$, rather than the correlation function. The condition (24) corresponds to extremely extended sojourn times in these states. For this reason, we think that an extremely good approximation to apply to $(19)$ is

$$
\sigma\left(x, t-t^{\prime}\right)=\frac{1}{2} \int_{-\infty}^{\infty} d x^{\prime} \delta\left(W t^{\prime}-\left|x-x^{\prime}\right|\right) \sigma\left(x^{\prime}, t\right) .
$$

For times $t^{\prime}$ such that $\left|t^{\prime}\right|\langle\langle t\rangle$, where $\langle t\rangle$ is the mean sojourn time in one of the two states, (26) is an exact property. The relation (26) becomes an approximation only for times larger than $\langle t\rangle$. We shall return to the discussion of $\langle t\rangle$ in Sec. IV.

By inserting (26) into (19) we obtain, with no approximation,

$$
\frac{\partial}{\partial t} \sigma(x, t)=\frac{1}{2} \frac{1}{W} \int_{-\infty}^{\infty} d x^{\prime} \Psi\left(\frac{\left|x-x^{\prime}\right|}{W}\right) \sigma\left(x^{\prime}, t\right),
$$


where the function $\Psi(t)$ is defined by means of

$$
\Psi(t)=\frac{\partial^{2}}{\partial t^{2}} \frac{\langle y y(t)\rangle}{W^{2}} .
$$

What is the physical meaning of $\Psi(t)$ ? Let us consider the function

$$
\psi(t) \equiv \frac{\Psi(t)}{\langle t\rangle} .
$$

This is a distribution of times. If we evaluate the mean value $\langle t\rangle$ averaging on this distribution we get

$$
\langle t\rangle=\frac{A^{1 / \beta}}{\beta}
$$

and $\psi(t)$ fits the normalization condition

$$
\int_{0}^{\infty} d t \psi(t)=1
$$

To assess the nature of this time distribution we use the relation established by Geisel and co-workers [27],

$$
\Phi_{y}(t)=\frac{1}{\langle t\rangle} \int_{t}^{\infty}\left(t^{\prime}-t\right) \psi_{s}\left(t^{\prime}\right) d t^{\prime}
$$

connecting the correlation function $\Phi_{y}(t)$ to the distribution of the sojourn times in the states $|+\rangle$ and $|-\rangle, \psi_{s}(t)$. By differentiating (32) twice we establish that

$$
\psi_{s}(t) \equiv \frac{\Psi(t)}{\langle t\rangle}
$$

and thus reach the conclusion

$$
\psi(t) \equiv \psi_{s}(t)
$$

Note that using (28) and (23), we can rewrite (27) as

$$
\frac{\partial}{\partial t} \sigma(x, t)=\frac{\beta(\beta+1) A}{2} \int_{-\infty}^{\infty} d x^{\prime} \frac{W^{1+\beta} \sigma\left(x^{\prime}, t\right)}{\left(A^{1 / \beta} W+\left|x-x^{\prime}\right|\right)^{\beta+2}} .
$$

This result (35) establishes a direct connection with the Lévy processes. Seshadri and West [28] have established that a centrosymmetric Lévy stable process, namely, a diffusion process with a distribution, the Fourier transform (characteristic function) of which is given by

$$
\hat{\sigma}(k, t)=e^{-b|k|^{\alpha} t}
$$

obeys the integro-differential equation

$$
\frac{\partial}{\partial t} \sigma(x, t)=b \frac{1}{\pi} \sin \left(\frac{\pi \alpha}{2}\right) \Gamma(1+\alpha) \int_{-\infty}^{\infty} d x^{\prime} \frac{\sigma\left(x^{\prime}, t\right)}{\left|x-x^{\prime}\right|^{\beta+2}} .
$$

Making the plausible assumption that the short-range region $\left|x-x^{\prime}\right| \approx A^{1 / \beta} W$ does not contribute to the long-time Lévy process, (35) becomes identical to a Lévy process with

$$
b=\frac{2 \pi \beta(\beta+1) A W^{\beta+1}}{\sin \left(\frac{\pi(\beta+1)}{2}\right) \Gamma(\beta+2)} .
$$

Note that at $\beta=1$, as it must be, the decay rate becomes proportional to $W^{2}$, which can be identified with the mean quadratic value of the "velocity" $y$. Throughout the whole anomalous diffusion regime $0<\beta<1$ the dacay rate depends on a fractional value of the "temperature." It is interesting to notice that (20) together with (23) leads to the asymptotic property:

$$
\lim _{t \rightarrow \infty}\left\langle x^{2}(t)\right\rangle=K t^{2 H}
$$

with

$$
H=1-\frac{\beta}{2}
$$

On the basis of this property one would be led to expect that the following rescaling will take place:

$$
\sigma(x, t)=\frac{1}{t^{\delta}} F\left(\frac{x}{t^{\delta}}\right), \quad t \rightarrow \infty,
$$

with

$$
\delta=H
$$

However, the theoretical results of this section indicate that the resulting diffusion process is of Lévy kind. Consequently, the correct rescaling is given by (41) with

$$
\delta=\frac{1}{\beta+1}
$$

The numerical results of Figs. 1 and 2 fully support this prediction. Note that the curves of Fig. 1 are plotted with respect to the variable $\xi \equiv x / t^{\delta}$, with $\delta=1 /(\beta+1)$, namely, by assuming the rescaling (43) to be true, whereas Fig. 2 refers to $\xi \equiv x / t^{\delta}$, with $\delta=H$, thus implying the rescaling (42) to hold. By inspection of these figures we see that the rescaling (42) is ruled out, whereas the rescaling (43) results in satisfactory agreement with the numerical results.

\section{TIME EVOLUTION OF THE CHARACTERISTIC FUNCTION}

A characteristic function is defined as

$$
C(t)=\left\langle e^{i k x}\right\rangle
$$

where \langle\rangle denotes the ensemble average. Thus the complex exponential must be averaged on the stationary distribution of the process, which is different from (36). In fact, the $\alpha$-stable Lévy process defined by (36) does not take into account the fact that the distribution must be truncated, namely, the distribution must be equal to zero for $x<-W t$ and $x>W t$. In Fig. 1 we see the result of a numerical simulation of the model (8) and (10). We see that the distributions are identical to what is predicted by (36) in the interval $-W t<x<W t$, but there are no tails outside this interval. 


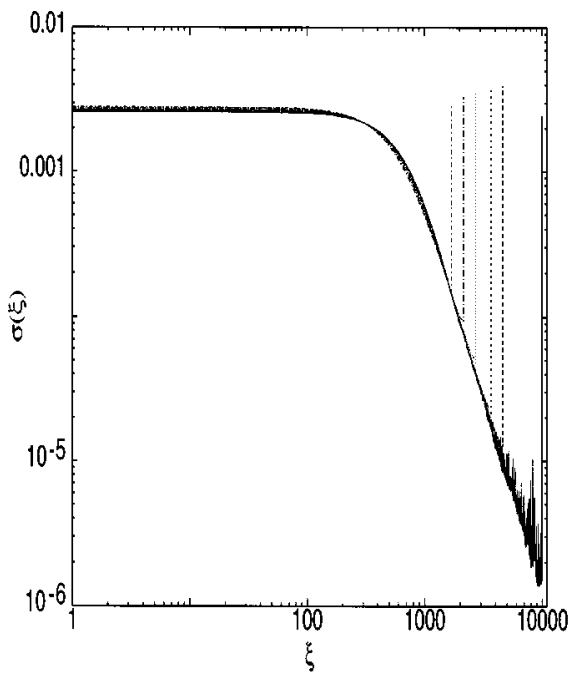

FIG. 1. Rescaled probability distributions for different times. The solid line refers to $t=10000$, the long-dashed line to $t=1000$, the short-dashed lineto $t=500$, the dotted line to $t=200$, the long-dash-dotted line to $t=100$, and the short-dash-dotted line to $t=50$. The distribution for $t=10000$ is not varied in this scale. The other ones have been rescaled according to $\delta=1 /(\beta+1)$.

These are substituted by ballistic peaks at $x= \pm W t$. The heigth of these peaks can be evaluated by considering them as the accumulation of the particles that would be in the tails if there was no ballistic limitation due to (8). One may think that asymptotically the peaks will vanish, but from the rescaled distributions of Fig. 1 we see that the ratio between their height and the height of the principal peak at $x=0$ is actually an increasing function of time. Asymptotically the peak height is

$$
\Lambda(t)=\int_{-\infty}^{-W t} \sigma(x, t) d x+\int_{W t}^{\infty} \sigma(x, t) d x
$$

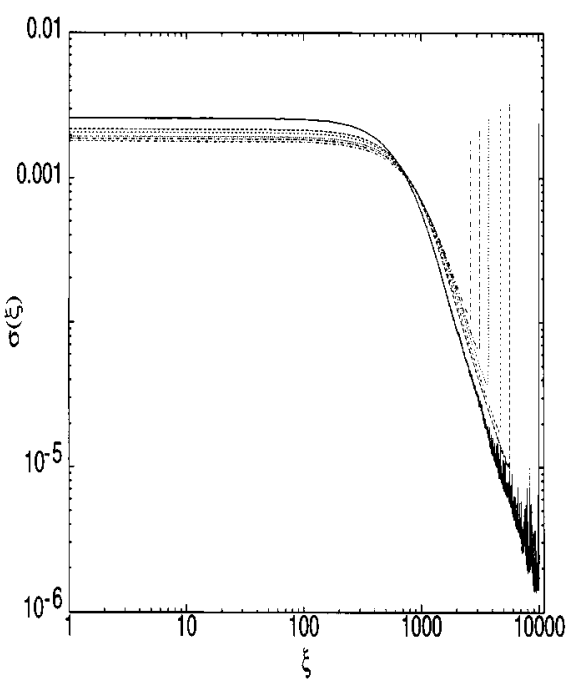

FIG. 2. Same as in Fig. 1, but the rescaling relation is given by $\delta=H=1-\beta / 2$. which can be proved to be proportional to the correlation function of the variable $y$. In fact, the first expansion of the Lévy distribution tails is $\sigma(x, t) \propto t /|x|^{\beta+2}$, so that (45) result in $\Lambda(t) \propto t^{-\beta}$. One can also think about the height of the peaks as the probability of finding a laminar region (namely, a region with all $y=W$ or $y=-W$ ) that is larger than $t$. Using the same arguments leading to (29) [27], it is possible to prove that this is just the correlation function, namely,

$$
\Lambda(t)=\Phi_{y}(t) .
$$

Now we can evaluate (44) by partitioning the integral in three pieces. We can write the total characteristic function as

$$
\begin{aligned}
C(t)= & \int_{-\infty}^{\infty} d x e^{i k x} \sigma(x, t)+\frac{\Lambda(t)}{2} \int_{-\infty}^{\infty} d x e^{i k x}[\delta(x+W t) \\
& +\delta(x-W t)]-2 \int_{W t}^{\infty} \frac{d x e^{i k x} t}{x^{\beta+2}}
\end{aligned}
$$

where the truncated Lévy process in the tails is substituted by the ballistic peaks. In the asymptotic regime the last member of the right-hand side of (47) is negligible. The first member is just the Fourier transform of $\sigma(x, t)$, which is given by (36), and the second member just gives $\Lambda(t) \cos (k t)$. Thus, for the characteristic function $C(t)$ we can write

$$
C(t)=e^{-b|k|^{\alpha} t}+\Phi_{y}(t) \cos (k t) ;
$$

notice that this form is not valid for short times. For $t<A^{1 / \beta}$, however, the peaks that are responsible for the second term on the rhs of (47) are the most important contribution and $C(t)=\cos (k t)$.

In Figs. 3(a)-3(d) we see the numerical results for the characteristic functions for different choices of the model parameters. We notice that in all cases the first regime is well fitted by a cosine function, while Eq. (48) also fits well for intermediate times. The asymptotic regime, in other words, is attained as soon as it is possible to make a continuous approximation to the distribution functions [see Fig. 3(a)]. The cosine behavior is the track of a dichotomous process, due to the fact that for times smaller than $\langle t\rangle$ the distributions $\sigma(x, t)$ are essentially dominated by the ballistic peaks. Notice that Eq. (48) can have a variety of behaviors: in particular, with a choice of a large $|k|$ value, which according to (6) means a strong-coupling condition, the first member on the rhs of (48) can be made negligible, so that the characteristic function reduces to $\Phi_{y}(t) \cos (k t)$ [see Fig. 3(b)]. On the other hand, a choice of very small $|k|$ may make the first term on the rhs of (48) the dominant one for the short- and intermediate-time regimes. This is especially true when $\beta$ is chosen near unity. In this latter case [see Fig. 3(c)] the characteristic function is almost indistinguishable from an exponential for all the times it can be computed. It has to be said that even in this case the small contribution of the second term on the rhs of (48) will sooner or later dominate since the cosine is modulated with a function that is nonintegrable, even though it has small numerical values. The property that the characteristic function in the short-time region is essentially a harmonic function of time, with negligible damping, is made evident by the results of numerical calculations by 

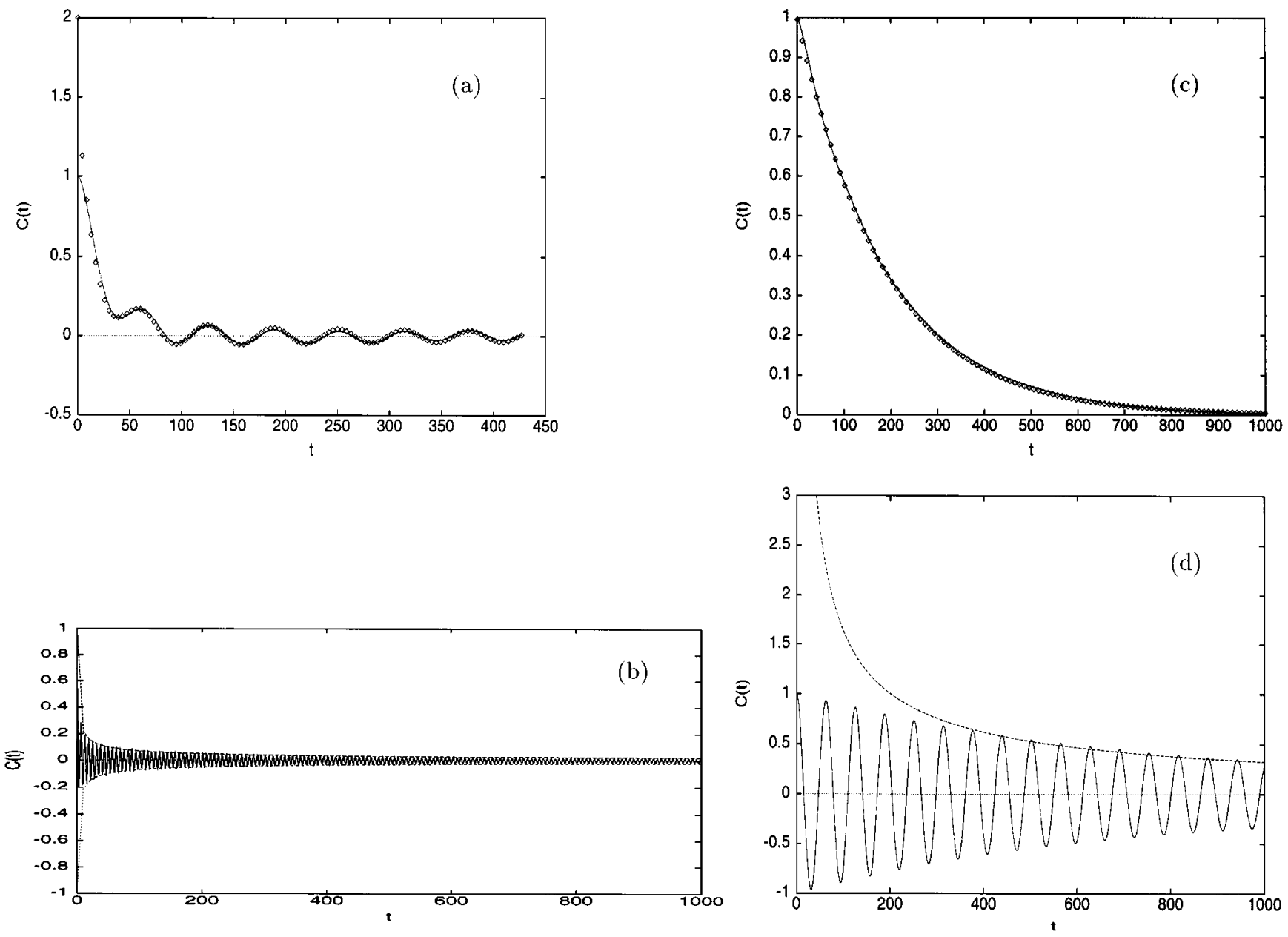

FIG. 3. Different characteristic functions for different parameter choices. (a) $\beta=0.5, k=0.1$, and $A$ is measured to be $\approx 0.5$ by the evaluation of the correlation function (not shown here). The solid line is the numerical evaluation of the characteristic function, while the dots are a sampling of the function (48) for the corresponding parameters. (b) $\beta=0.5, k=1.0$, and $A \approx 0.5$. The solid line represents the numerical evaluation of the characteristic function. The two dotted lines over and under the curve are the correlation function (23) and its opposite. (c) $\beta=0.99, k=0.05$, and $A \approx 1$ : same as in (a). (d) $\beta=0.7, k=0.1$, and $A \approx 41.1$. The solid line represent the numerical evaluation of the characteristic function, while the upper dashed curve is $A / t^{\beta}$.

making suitably extended this initial time region. This can be done by using very large values of $A$, which mean very large values of $\langle t\rangle$. This condition is conveniently illustrated by Fig. 3(d).

\section{CONCLUDING REMARKS}

We have seen, probably in a way more transparent than in earlier work [13], that the dynamical realization of Lévy process with a velocity $y$, with fluctuations whose intensity cannot exceed a given upper value, implies that the resulting diffusion equation is strongly non-Markovian. The realization of a Lévy process, which is by definition Markovian, implies that the time nonlocality is turned into a space nonlocality. This is made possible by means of the constraint (26), which is based on the fact that for a long period of time the motion takes place without any change of the velocity value. It is interesting that Compte recently observed [29] that the adoption of the continuous-time random-walk method is essentially equivalent to adopting a generalized, namely non-Markovian, master equation. The above analysis makes it easier to understand a further result of Compte [29], namely, that it is possible to express the long-time regime in two equivalent forms, one implying the adoption of the fractional time derivative (and so emphasizing time nonlocality) and the other a fractional space derivative (and so emphasizing the space nonlocality or the Lévy nature of the statistical process [28]).

It has to be pointed out, however, that the approach presented in this paper rests only on dynamical properties and makes it possible, in principle, to derive the resulting Lévy process directly from the equation of motion of the system. This was already done $[6,14-16]$ in the case of ordinary diffusion, but all the recent papers on the diffusion processes faster than ordinary diffusion $[11,13]$ still derive the Lévy statistics from probabilistic assumptions rather than using only dynamical properties. Thus the key statistical feature of all these treatments is the distribution of waiting times in the two states of the dichotomous variable $\psi(t)$. Herein, on the contrary, all the relevant statistical properties of the diffusion process are expressed in terms of the correlation function $\Phi_{y}(t)$, thus establishing a more direct dependence on the dynamics of the system. 
We have to point out that the inverse power-law nature of the correlation function $\Phi_{y}(t)$, the asymptotic property stemming from (23), is assumed rather than derived from the equations of motion of the system. However, this is where our approach has some merit when compared to the current literature on the derivation of the Lévy statistics. On the one hand, the current theoretical treatments of superdiffusion rests on the inverse power law nature of $\psi(t)$ [11-13]. On the other hand, there are theoretical approaches to the inverse power law of the waiting function distribution $\psi(t)$ [30], which in the asymptotic time limit is proportional to $t^{-\mu}$. However, the numerical check of these theoretical predictions for dynamical systems such as the kicked rotator is made difficult by the fact that the computational derivation of $\psi(t)$ is much less accurate than the second moment of the diffusing variable as a function of time [compare, for instance, the function $\psi(t)$ as numerically derived in [12] to the second moment of the angular momentum of the kicked rotator in [31]]. Using our approach the exponent $H$ of the time asymptotic behavior of the second moment of $x$ [see Eq. (20)] is immediately related to the asymptotic properties of the correlation function $\langle y y(t)\rangle$ and through (28), (33), and (34) to the inverse power law of $\psi(t)$ with $\mu=4-2 H$. This relation and the close connection with the Lévy statistics seem to be more direct, more concise, and more satisfactory than any earlier derivation. We think that it also explains why the velocity model of Zumofen and Klafter [9], resting on (32), turns out [13] to be the best theoretical approach to superdiffusion based on random walk arguments.

From the point of view of applications, the method of this paper affords further benefits, especially if it is applied to problems of biological significance such as that of the DNA sequences [32]. A direct calculation of $\psi(t)$ in this case from the experimental data would lead us to define the $\psi(t)$ corresponding to the two-state model of Ref. [9] rather than to the velocity model of the same authors. This would make ambiguous the analysis of the data in this case, since the existence of a dynamical model underlying the DNA sequences is a conjecture rather than a established fact [32] and if this dynamical model exists its nature is unknown. If the theory developed in this paper is adopted, it is possible to carry out the analysis of the DNA sequences with no ambiguity. Using the prescriptions of Ref. [32] we can evaluate the correlation function $\Phi_{y}(t)$, thereby providing a dynamical basis for Lévy statistics found herein.

A further result of this paper is that, in spite of the fact that the asymptotic regime of the distribution is very close to Lévy statistics, the study of the characteristic function might reveal the strongly non-Markovian nature of the process, as effectively expressed by (48). Of special interest is the fact that even if for $\beta$ very close to unity, or small values of $|k|$ (implying a very weak coupling between the precessing dipole and the fluctuating "magnetic" field), an almost exact exponential behavior is obtained, still the slow tails with $\beta<1$ are present. This agrees with the important result of Lee [33] establishing that a decay process cannot be an exact exponential and shows that the dynamical derivation of Lévy processes is compatible with a rigorous Hamiltonian treatment.

Finally, we want to point out that the results of this paper might have some interest for the problem of the macroscopic manifestation of quantum mechanics. According to Zurek [34] a genuinely irreversible decoherence process must be perceived as being totally equivalent to a wave function collapse, thereby allowing quantum mechanics to recover classical mechanics with no need of changing the current version of this theory. However, this raises two problems. The first has to do with the earlier mentioned hot issue of how to derive a rigorously exponential behavior from within ordinary quantum mechanics. Figure 3(c) shows that the identification of the almost exponential decay of the characteristic function might be questionable since the exponential-like behavior is followed by an invisible slow tail implying the presence of correlation even after the occurrence of a wavefunction collapse. The second problem has to do with the fact [35] that the theory of Zurek rests on the assumption that the environment is faithfully described by ordinary statistical mechanics. The results of Sec. IV show that as a consequence of rejecting this assumption, the decorrelation process might become extremely slow, thereby supporting the requirement that the environment is driven by ordinary statistical mechanics. On the other hand, the dynamical erivation of ordinary statistical mechanics is made possible by the assumption of time-scale separation between macroscopic and microscopic variables [6] and this paper can be seen as an attempt at extending this treatment to the case where the time-scale separation is not possible.

\section{ACKNOWLEDGMENT}

The authors thank the Office of Naval Research for partial support of this research.

\section{APPENDIX}

The numerical calculations adopted in this paper to check the theoretical predictions rest on a dynamical process that is not generated by a weakly chaotic deterministic map as in Refs. $[9,13]$. The possibility of generating a correlation function with the same time asymptotic properties as in (23) is now well established not only in the case of the map of Geisel, Nierwetberg, and Zacherl [36], but also in the case of the kicked rotator in the so called accelerating state $[12,37]$. However, the direct use of a deterministic map to assess numerically the time asymptotic properties implies a significant computational effort in terms of both computer time and numerical precision. For this reason, we decided to adopt a different procedure. First of all, the present treatment rests directly on the correlation function $\Phi_{y}(t)$ rather than indirectly through the waiting-time distribution $\psi(t)$. However (28) and (32) show that the two descriptions are equivalent. To generate a dichotomous process with a given $\Phi_{y}(t)$ it is sufficient to generate a random sequence of integers with a probability distribution given by a discrete approximation of

$$
\psi(t)=\frac{(\beta+1) A^{(\beta+1) / \beta}}{\left(A^{1 / \beta}+t\right)^{\beta+2}} ;
$$

this can be done through a filtered random number generator. In other words, our dichotomous sequence is given by randomly choosing a number between +1 and -1 and keeping the same value for $y$ a number of times that is given by our filtered random number generator. Then the same operation 
is repeated until the statistics are sufficient for the calculation. Care is exerted to ensure that at the end of the period of time spent in one state of the velocity $y$ the probability of jumping from one to the other state is the same as that of remaining in the same state. Under this condition (32) is an exact relation and building up the waiting-time distribution $\psi(t)$ (49) turns out to be equivalent to building up the correlation function $\Phi_{y}(t)(23)$.

The dynamical generator used is therefore not determin- istic. However, the main point to assess in this paper refers to the physical consequences of a dynamical generator responsible for the inverse-power-law character of (23). Once this is determined, the results are independent of whether the dynamical generator has a deterministic or a stochastic origin. Thus, for numerical convenience we have adopted the second type of dynamical generator. This method of numerical calculation turned out to be so efficient as to result in the very regular curves denoted by the solid lines of Fig. 3 .
[1] N.G. van Kampen, Stochastic Processes in Physics and Chemistry (North-Holland, Amsterdam, 1981).

[2] H. Mori, H. Hata, T. Horita, and T. Kobayashi, Suppl. Prog. Theor. Phys. 99, 1 (1989).

[3] K. Lindenberg and B.J. West, The Nonequilibrium Statistical Mechanics of Open and Closed Systems (VCH New York, 1990).

[4] E. Ott, Chaos in Dynamical Systems (Cambridge University Press, Cambridge, 1993).

[5] B.V. Chirikov, Phys. Rep. 52, 263 (1979).

[6] M. Bianucci, R. Mannella, B. J. West, and P. Grigolini, Phys. Rev. E 51, 3002 (1995).

[7] E. W. Montroll and B.J. West, in Fluctuation Phenomena, 2nd ed., edited by E.W. Montroll and J. L. Levowitz, Studies in Statistical Mechanics Vol. 7 (North-Holland, Amsterdam, 1987); E.W. Montroll and M.F. Shlesinger, in From Stochastics to Hydrodynamics, edited by J. L. Lebowitz and E. W. Montroll (North-Holland, Amsterdam, 1984).

[8] M.F. Shlesinger, B.J. West, and J. Klafter, Phys. Rev. Lett. 58, 1100 (1987).

[9] G. Zumofen and J. Klafter, Phys. Rev. E 47, 851 (1993).

[10] See, for example, M.F. Shlesinger, G. M. Zaslavski, and J. Klafter, Nature 363, 31 (1993).

[11] G. Zumofen and J. Klafter, Physica A 196, 102 (1993); Physica D 69, 436 (1993).

[12] J. Klafter, G. Zumofen, and M.F. Shlesinger, Fractals 1, 389 (1993); Europhys. Lett. 25, 565 (1994).

[13] G. Trefan, E. Floriani, B.J. West, and P. Grigolini, Phys. Rev. E 50, 2564 (1994).

[14] R. V. Jensen, Phys. Rev. A 30, 386 (1984).

[15] T. Geisel and S. Thomae, Phys. Rev. Lett 52, 1936 (1984).

[16] S. Grossman and H. Fujisaka, Phys. Rev. A 26, 1779 (1982)

[17] P. Garbaczewski, in Chaos-The Interplay Between Stochastic and Deterministic Behaviour, edited by P. Garbaczewski, M. Wolf, and A. Weron (Springer-Verlag, Berlin, 1995).

[18] C. Beck, in Chaos-The Interplay Between Stochastic and Deterministic Behaviour (Ref. [17]), p. 3.

[19] W.G. Glockle and T.F. Nonnenmacher, Macromolecules 24, 6426 (1991).
[20] H. Schiessel, R. Metzler, A. Blumen, and T.F. Nonnenmacher, J. Phys. A 28, 6567 (1995).

[21] W.G. Glockle and T.F. Nonnenmacher, J. Stat. Phys. 71, 741 (1993).

[22] P. Grigolini, V. M. Kenkre, and D. Vitali, Phys. Rev. A 43, 1015 (1991).

[23] A. S. Davydov and N. I. Kislukha, Phys. Status Solidi (B) 59, 465 (1973); A. S. Davydov, Usp. Fiz. Nauk SSSR 138, 603 (1982) [Sov. Phys. Usp. 25, 898 (1982)]; Biology and Quantum Mechanics (Pergamon New York, 1982).

[24] V. M. Kenkre and D. K. Campbell, Phys. Rev. B 34, 4595 (1986); V. M. Kenkre and H.-L. Wu, ibid. 39, 6907 (1989); Phys. Lett. A 135, 120 (1989).

[25] A. J. Leggett, S. Chakravarty, A. T. Dorsey, M. P. A. Fisher, A. Garg, and W. Zwerger, Rev. Mod. Phys. 59, 1 (1987).

[26] W. Horsthemke and R. Lefever, Noise Induced Transitions, Theory and Applications in Physics, Chemistry and Biology (Springer, Berlin, 1984).

[27] T. Geisel, J. Heldstab, and H. Thomas Z. Phys. B 55, 165 (1984).

[28] V. Seshadri and B. J. West, Proc. Natl. Acad. Sci. U.S.A. 79, 4051 (1982).

[29] Compte, Phys. Rev. E 53, 4191 (1996).

[30] J. D. Meiss and E. Ott, Phys. Rev. Lett. 55, 2741 (1985); Physica D 20, 387 (1986).

[31] R. Ishizaki, T. Horita, T. Kobayashi, and H. Mori, Prog. Theor. Phys. 85, 1013 (1991).

[32] P. Allegrini, M. Barbi, P. Grigolini, and B.J. West, Phys. Rev. E 52, 5281 (1995).

[33] H. Lee, Phys. Rev. Lett. 51, 1227 (1983).

[34] W. H. Zurek, Phys. Rev. D 24, 1516 (1981); 26, 1862 (1982); Phys. Today 44 (10), 36 (1991).

[35] P. Grigolini, in Chaos-The Interplay between Stochastic and Deterministic Behaviour (Ref. [17]), p. 101.

[36] T. J. Geisel, J. Nierwetberg, and A. Zacherl, Phys. Rev. Lett. 54, 616 (1985).

[37] T. Horita, H. Hata, R. Ishizaki, and H. Mori, ibid. 83, 1065 (1990); R. Ishizaki, H. Hata, T. Horita, and H. Mori, Prog. Theor. Phys. 84, 179 (1990). 